\title{
Reduction of Current Ripple and Acoustic Noise in Dual-inverter Pole-changing Induction Motor Drives
}

\author{
K.T. Chau, S.Z. Jiang and C.C. Chan \\ Dept. of Electrical \& Electronic Engineering, University of Hong Kong, Hong Kong, China
}

\begin{abstract}
In this paper, a new dual-inverter pole-changing induction motor drive is proposed and analyzed. The two keys are to adopt a new pole-changing induction motor to extend the constant-power operating range and to develop a new dualinverter to significantly suppress the harmonic torques (hence the acoustic noise). The correspondingly maximum torque capability and harmonic torque cancellation are particularly analyzed and discussed.
\end{abstract}

\section{INTRODUCTION}

The squirrel cage induction motor (SCIM) is one of the most viable motors for electric vehicle (EV) propulsion [1]. There are two major challenges for its application to EV propulsion: (1) difficulty in providing constant-power operation for the high-speed range of EVs; (2) acoustic noise problem throughout the wide-speed range of EVs.

The first challenge has been alleviated by using fluxweakening control during constant-power operation. Nevertheless, the corresponding SCIM can only provide constant-power operation up to about twice the base speed, which is far below the desired constant-power speed range (four to five times the base speed) for EVs. In order to realize such high-speed constant-power operation, additional strategies such as increasing the maximum torque of the motor or the voltage level of the battery may be adopted, but suffering from the problem of large motor size or heavy battery weight. One possible way to solve this problem is to adopt a large pole number for high-torque low-speed operation, and then to automatically change to a small pole number for constant-power high-speed operation. Therefore, the inverter-fed pole-changing SCIM (characterized by sixphase windings and dual inverters) is becoming attractive for modern EVs [2].

The second challenge has been alleviated by using new PWM algorithms, especially the random PWM [3]. By using this random PWM algorithm, the carrier frequency and hence the acoustic peak noise are randomly distributed so that the overall acoustic noise level can be significantly reduced. Nevertheless, one key drawback of this random PWM is the possibility to create the disastrous mechanical resonance between the randomized harmonic torques and the mechanical natural vibration frequencies [4].

In this paper, both two major challenges on the use of SCIM for EV propulsion are simultaneously solved in a new way. Namely, a new six-phase pole-changing SCIM is fed by dual three-phase inverters in such a way that the harmonic torques and hence the acoustic noise are suppressed.

\section{SIX-PHASE SCIM}

Fig. 1 shows the proposed dual-inverter pole-changing SCIM. The original three-phase windings $(\mathrm{A}-\mathrm{B}-\mathrm{C})$ are split into two sets of three-phase windings (a-b-c and $d-e-f)$, namely dual-star six-phase windings. There are 36 slots in the stator of the designed motor. All 36 coils are of double layers. The distribution of these coils is shown in Table $I$ that illustrates how the six-phase windings evolved from the three-phase windings. The corresponding winding connection is illustrated in Fig. 2. For instance, the phase A winding consists of two sets of windings, namely $a-O$ and $d-O$, and each of them is composed of six coils. The coil connections of the phase $\mathrm{B}$ and $\mathrm{C}$ windings are formed in a similar way.

Fig. 3 shows the MMF waveforms at the instant that the current of the phase A winding reaches its maximum value.

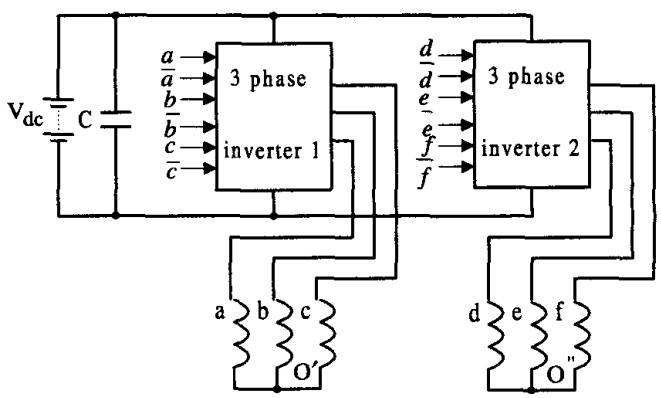

Fig. 1. Dual-inverter pole-changing SCIM.

TABLE I

COIL DISTRIBUTION

\begin{tabular}{|c|c|c|c|c|c|c|c|c|c|}
\hline number & 1 & 2 & 3 & 4 & 5 & 6 & 7 & 8 & 9 \\
\hline 4 poles & $\bar{A}$ & A & $\overline{\mathrm{A}}$ & $\mathrm{C}^{-}$ & $\mathrm{C}^{-}$ & $\mathrm{C}^{-}$ & B & B & $\bar{B}$ \\
\hline 8 poles & A & $\mathbf{A}$ & A & C & C & C & B & B & B \\
\hline 6-phase & a & a & $\mathbf{a}$ & f & f & f & $b$ & b & b \\
\hline number & 10 & 11 & 12 & 13 & 14 & 15 & 16 & 17 & 18 \\
\hline 4 poles & $\mathrm{A}^{\prime}$ & $\mathbf{A}^{-}$ & $\mathrm{A}^{\circ}$ & C & C & $\mathrm{C}$ & $\mathrm{B}^{-}$ & $\mathrm{B}^{-}$ & $\mathbf{B}^{-}$ \\
\hline 8 poles & $\mathbf{A}$ & $\mathbf{A}$ & A & C & C & C & B & B & B \\
\hline 6-phase & d & d & $\mathrm{d}$ & $\mathrm{c}$ & $\mathrm{c}$ & c & e & $\mathrm{e}$ & e \\
\hline number & 19 & 20 & 21 & 22 & 23 & 24 & 25 & 26 & 27 \\
\hline 4 poles & $\mathrm{A}$ & $\mathbf{A}$ & A & $\mathrm{C}^{-}$ & $\mathrm{C}^{\circ}$ & $\mathrm{C}^{-}$ & B & B & B \\
\hline 8 poles & A & A & A & C & C & C & B & B & B \\
\hline 6-phase & a & a & a & f & f & $\mathrm{f}$ & b & b & b \\
\hline number & 28 & 29 & 30 & 31 & 32 & 33 & 34 & 35 & 36 \\
\hline 4 poles & $\mathrm{A}^{-}$ & $\mathrm{A}^{-}$ & $\mathrm{A}^{\circ}$ & $\mathrm{C}$ & $\mathrm{C}$ & $\mathrm{C}$ & $\mathrm{B}^{-}$ & $\mathrm{B}^{-}$ & $\mathrm{B}^{-}$ \\
\hline 8 poles & A & A & A & C & C & C & B & B & B \\
\hline 6-phase & $\mathrm{d}$ & d & d & $\mathrm{c}$ & c & c & e & e & $\mathbf{e}$ \\
\hline
\end{tabular}




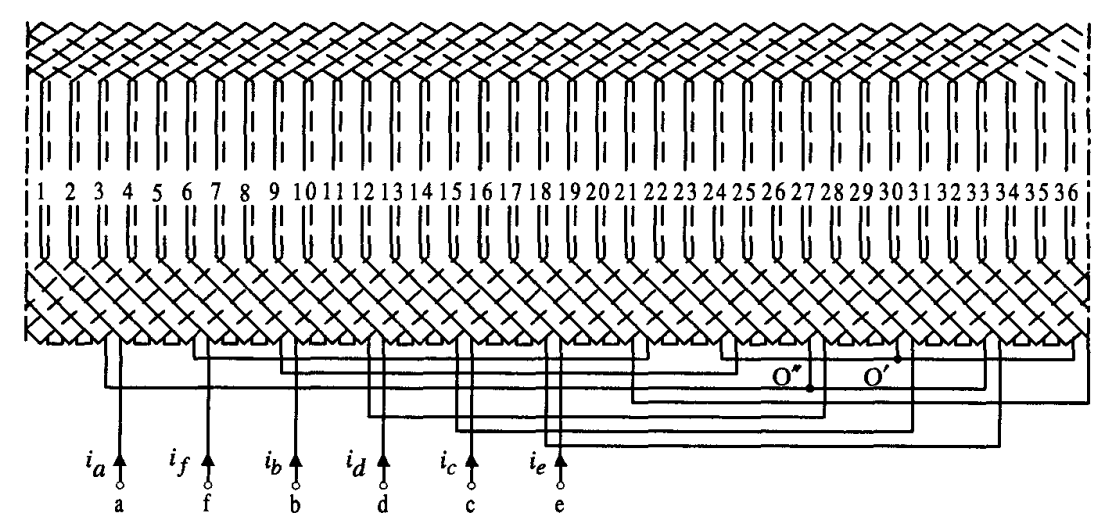

Fig. 2. Winding connection.

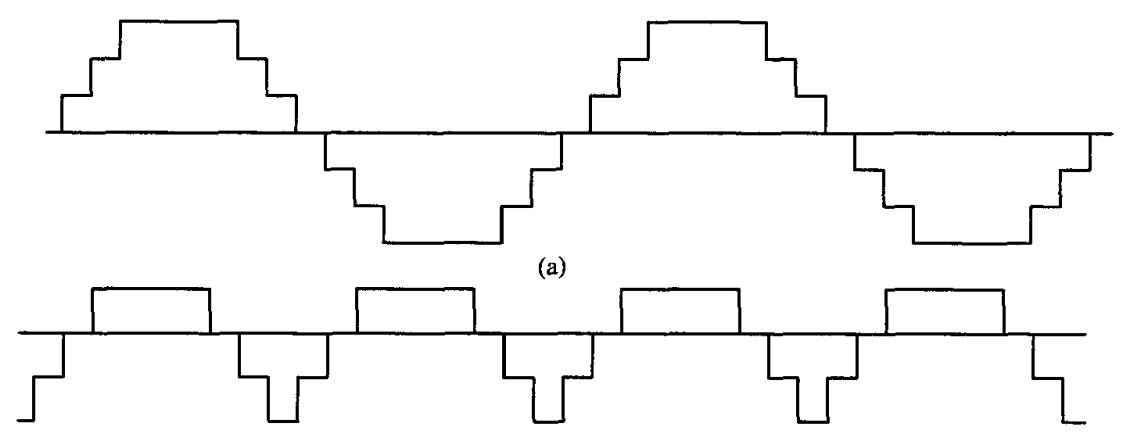

(b)

Fig. 3. MMF waveforms. (a) 4-pole. (b) 8-pole.

In Fig. 3(a), the currents of two star-connected windings are out of phase, producing a 4-pole MMF waveform. In Fig. 3 (b), the two currents become in phase, producing the 8-pole MMF waveform. Thus the number of poles can be altered by changing the phase difference between the currents of two star-connected windings. Hence by controlling the switching signals of the dual inverters for this six-phase SCIM, the corresponding pole pairs can be changed electronically.

\section{MAXIMUM TORQUE CAPABILITY}

The dual-star connected six-phase windings can be considered as three-phase windings, consisting of the windings $a$ and $d, b$ and $e$ as well as $c$ and $f$. The per-phase equivalent circuits for 4-pole and 8-pole operation are shown in Fig. 4. Thus the calculation method of the maximum torque is the same as that of a three-phase SCIM. The ratio of the 4-pole maximum torque $T_{4 m}$ to the 8-pole maximum torque $T_{8 m}$ is given by:

$$
\begin{aligned}
\frac{T_{4 m}}{T_{8 m}}= & \frac{p_{4}}{p_{8}} \frac{f_{8}}{f_{4}}\left(\frac{2 U_{4}}{U_{8}}\right)^{2} \\
& \frac{0.5 R_{s}+\sqrt{\left(0.5 R_{s}\right)^{2}+\left(0.5 X_{8 s}+0.5 X_{8 r}^{\prime}\right)^{2}}}{2 R_{s}+\sqrt{\left(2 R_{s}\right)^{2}+\left(2 X_{4 s}+0.5 X_{4 r}^{\prime}\right)^{2}}}
\end{aligned}
$$

where the subscripts 4 and 8 respectively indicate the 4-pole and 8-pole variables, $p_{4}$ and $p_{8}$ are the number of pole pairs, $U_{4}$ and $U_{8}$ are the phase voltages, $f_{4}$ and $f_{8}$ are the supply frequencies, $R_{s}$ is the stator resistance, $X_{4 s}$ and $X_{8 s}$ are the stator leakage reactances, $X_{4 r}^{\prime}$ and $X_{8 r}^{\prime}$ are the referred rotor leakage reactances.

During constant-power operation, the phase voltage reaches its maximum value, namely $U_{4}=U_{8}$. Due to high operating frequency, $R_{s}$ is negligible when compared with the leakage reactances. Since these leakage reactances are proportional to the frequency, while $p_{8}=2 p_{4}$ and $f_{8}=2 f_{4}$, (1) can be rewritten as:

$$
\frac{T_{4 m}}{T_{8 m}}=2 \cdot \frac{2 l_{s}+2 k_{8}^{2} l_{r}}{2 l_{s}+0.5 k_{4}^{2} l_{r}}
$$

where $l_{s}$ is the stator leakage inductance, $l_{r}$ is the rotor leakage inductance, $k_{8}$ is the 8-pole operation referred factor and $k_{4}$ is the 4-pole operation referred factor. The referred factors can be expressed as:

$k_{8}=\frac{24\left(T_{s} k_{w 8}\right)^{2}}{N_{r}}$ 


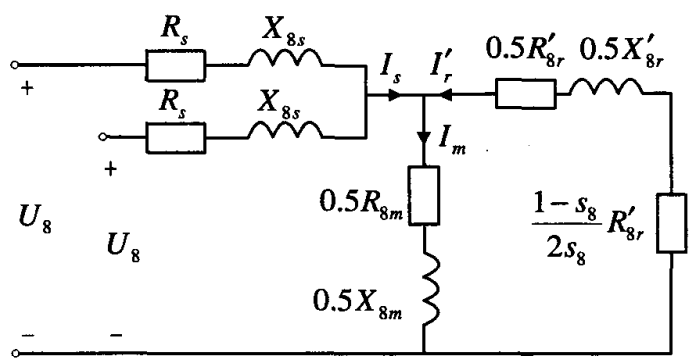

(a)

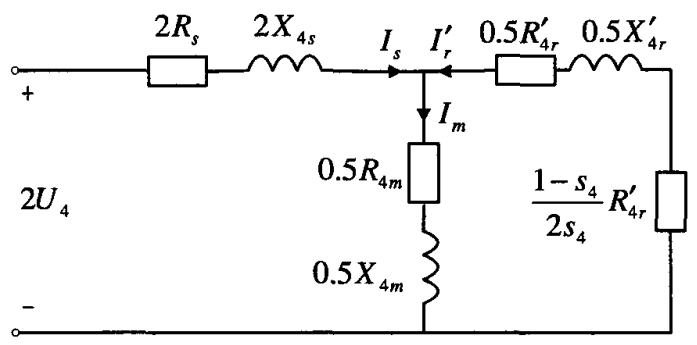

(b)

Fig. 4. Equivalent circuits. (a) 8-pole. (b) 4-pole.

$k_{4}=\frac{24\left(T_{s} k_{w 4}\right)^{2}}{N_{r}}$

where $T_{s}$ is the number of turns in series per phase, $N_{r}$ is the number of rotor slots, $k_{w 8}$ is the 8-pole winding factor and $k_{w 4}$ is the 4-pole winding factor.

During start-up or low-speed operation, the operating frequency is so low that the leakage reactances can be neglected when compared with $R_{s}$. In order to keep the saturation level in the yoke constant, the air-gap flux density at 4-pole operation is half of that at 8-pole operation. Thus, the ratio of voltages can be obtained as:

$\frac{U_{4}}{U_{8}}=\frac{f_{4}}{f_{8}} \frac{k_{w 4}}{k_{w 8}}$

By using (5), (1) can be rewritten as:

$\frac{T_{4 m}}{T_{8 m}}=\frac{1}{4}\left(\frac{k_{w 4}}{k_{w 8}}\right)^{2}$

During normal subsynchronous speed operation, (5) is still valid and (1) can be rewritten as:

$$
\begin{aligned}
\frac{T_{4 m}}{T_{8 m}}= & \left(\frac{k_{w 4}}{k_{w 8}}\right)^{2} \\
& \frac{0.5 R_{s}+\sqrt{\left(0.5 R_{s}\right)^{2}+\left(0.5 X_{8 s}+0.5 X_{8 r}^{\prime}\right)^{2}}}{2 R_{s}+\sqrt{\left(2 R_{s}\right)^{2}+\left(2 X_{4 s}+0.5 X_{4 r}^{\prime}\right)^{2}}}
\end{aligned}
$$

With increasing frequency, the leakage reactances are approaching $R_{s}$. Assuming that $X_{8 s}=X_{8 r}^{\prime}=R_{s}$ and $X_{4 s}=X_{4 r}^{\prime} \approx 0.5 R_{s},(7)$ can be simplified as:
$\frac{T_{4 m}}{T_{8 m}} \approx \frac{1}{2.7}\left(\frac{k_{w 4}}{k_{w 8}}\right)^{2}$

In (6), if the winding factors satisfy the inequality:

$$
k_{w 8}>k_{w 4}
$$

the 8-pole maximum torque is more than four times the 4pole maximum torque.

In (2), provided that the relation:

$2 k_{8}^{2} \geq 0.5 k_{4}^{2}$

is satisfied, the 4-pole maximum torque doubles the 8-pole maximum torque in the constant-power operation range. Applying (3) and (4) to (10), it results in

$k_{w 8} \geq 0.5 k_{w 4}$

From (9) and (11), as long as (9) is satisfied, (11) can also be satisfied correspondingly. Hence the maximum torque characteristic of pole-changing SCIM shown in Fig. 5 can be obtained. The position of the intersection depends on the ratio of winding factors. The larger the ratio is, the longer the intersection is away from the origin. For the proposed SCIM, the winding distribution of the double layer $60^{\circ}$ phase spread is employed in 4-pole operation, while 8-pole operation results in $120^{\circ}$ phase spread. Table II shows the winding factors under different coil pitches ( $N_{P}$ is the coil pitch). It can be found that the constraint given by (9) is easy to be satisfied. In addition, the maximum torque in certain pole pairs is subjected to the winding factor and a large winding factor produces a higher maximum torque. Hence the winding factor should be determined to conform the load characteristic of EV.

\section{DUAL-INVERTER OPERATION}

In each three-phase inverter, SPWM algorithm is adopted. Its switching signal is generated by comparing three-phase

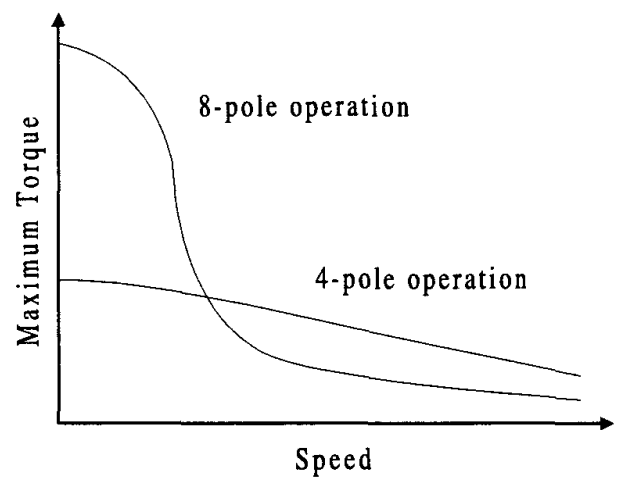

Fig. 5. Maximum torque characteristic of pole-changing SCIM

TABLE II

WINDING FACTORS

\begin{tabular}{|c|c|c|c|c|c|c|c|}
\hline$N_{P}$ & 3 & 4 & 5 & 6 & 7 & 8 & 9 \\
\hline$k_{w 8}$ & 0.731 & 0.831 & 0.831 & 0.731 & 0.542 & 0.289 & 0 \\
\hline$k_{w 4}$ & 0.48 & 0.616 & 0.735 & 0.831 & 0.901 & 0.945 & 0.961 \\
\hline
\end{tabular}


symmetrical sinusoidal reference of variable amplitude with a triangular carrier. For 8-pole operation, the phases and amplitudes of the two sets of three-phase references are identical. Under this condition, the phase between two triangular carriers leads to two possible cases on how to generate the PWM signals - namely, the case 8P1 for which the two carriers are in phase, and the case $8 \mathrm{P} 2$ for which the two carriers are exactly out of phase. On the other hand, for 4-pole operation, the phases between the two sets of threephase references are exactly out of phase while their amplitudes are kept the same. Correspondingly, there are also two possible cases - namely, 4P1 and 4P2 for which the phases between two carriers are in phase and exactly out of phase respectively.

As shown in Fig. 1, dual voltage source PWM inverters are adopted for computer simulation. The frequency of the two carriers, $f_{c}$, is selected as $2 \mathrm{kHz}$. Assume that the fundamental frequency of 8-pole operation is $50 \mathrm{~Hz}$ and the modulation index is 0.8 . Under the same speed, the fundamental frequency of 4-pole operation is $25 \mathrm{~Hz}$. In order to keep the yoke flux density unchanged, the ratio of modulation indices should be equal to that of voltages given by (5). From Table II, the coil pitch is selected as 5 , hence the modulation index of 4-pole operation is 0.452 . Generally speaking, $f_{c}$ is fixed and the ratio of $f_{c}$ to $f$ (fundamental frequency) varies with $f$. Hence, both odd and even orders of harmonic voltages have chances to present in the phase voltage [5]. Because the windings are connected into dual stars, the triplen harmonic voltages cannot produce currents in the windings. These harmonic voltages are not considered.

The Fourier analysis results of the phase voltages are shown in Figs. 6 to 8. Fig. 6 shows the distribution of the amplitudes of the harmonic phase voltages in all four cases. The harmonic amplitudes at the integer multiples of the carrier frequency are larger than those nearby them. For the case $8 \mathrm{P} 1$, the amplitudes of the fundamentals and harmonics in the outputs of the inverter 1 and 2 are identical. For the case $8 \mathrm{P} 2$, since the phase of the carrier used in the inverter 2 is opposite to that in inverter 1 , the amplitudes are different from those in the case 8P1. But, since the fundamental and harmonics with large amplitudes change slightly, they are approximately equal. Since the fundamental amplitude varies in proportion to the modulation index, the amplitudes of the fundamentals and harmonics in the cases 4P2 and 4P1 are 0.565 times those in the cases $8 \mathrm{P} 1$ and $8 \mathrm{P} 2$ respectively. The phase differences between the harmonic voltages of phase a and phase $d$ are also the phase differences between two sets of symmetrical three-phase voltages. Relative to the phase of the fundamental voltage in the inverter 1 , the harmonic voltage phases of phase a and phase $d$ are shown in Figs. 7 and 8 . In the case $8 \mathrm{P} 1$, the fundamental and all harmonics of phase a are in phase with that of phase $d$. In the case 8P2, the even order harmonics of phase a and phase $d$ become out of phase. In the case 4P2, the fundamental and harmonic voltages of phase a and phase $d$ are out of phase. In case 4P1,

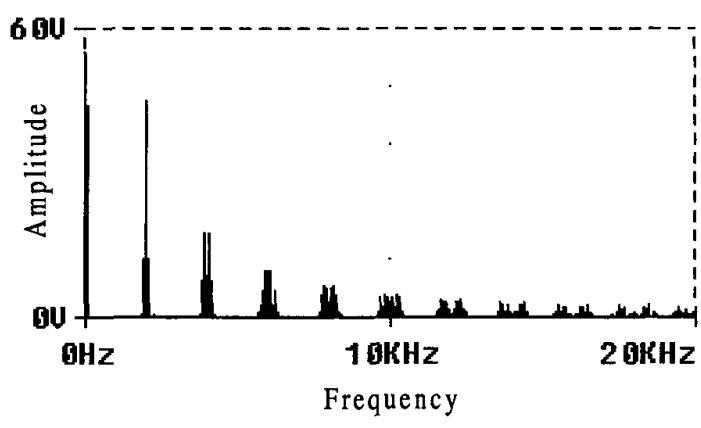

Fig. 6. Voltage amplitude spectrum.

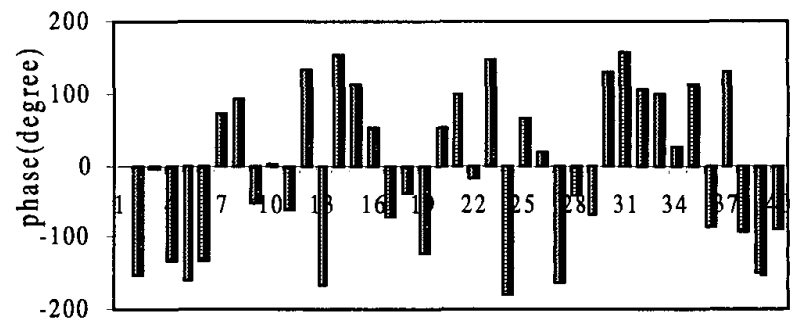

(a)

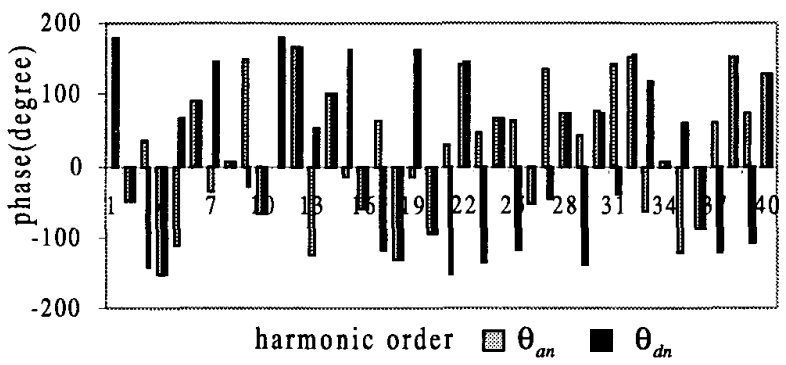

(b)

Fig. 7. Phase of fundamental and harmonic voltages .(a) 8P1. (b) 8P2.

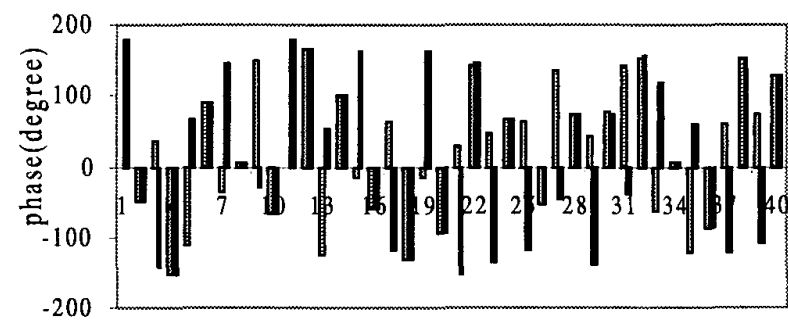

(a)

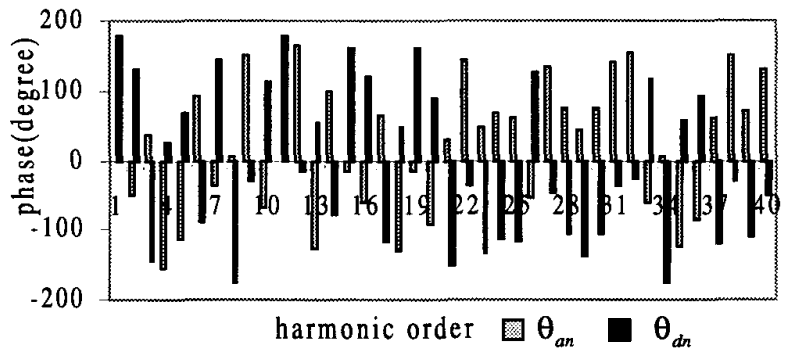

(b)

Fig. 8. Phase of fundamental and harmonic voltages. (a) 4P1. (b) 4P2. 
the even order harmonic voltages become in phase.

If the saturation effect is neglected, the fundamental and harmonic voltages can be regarded as independent voltage sources. Based on the simplified equivalent circuits shown in Fig. 9, the fundamental and harmonic currents can be calculated. The current waveforms of phase a and $d$ are shown in Figs. 10 and 11. They testify that the number of pole pairs is only dependent on the phase between references, irrelevant to the phase of carriers. It can be anticipated that the current amplitude spectrum is similar to the voltage amplitude spectrum shown in Fig. 6. The harmonic voltages and currents of the order $3 n-1$ ( $n$ is an integer) are negative sequence harmonics, whereas the $(3 n+1)$ th are positive sequence harmonics. The corresponding phasor diagrams are illustrated in Fig. 12.

The MMF and air-gap flux waves produced by the fundamental and harmonics rotate at different speeds and directions. Their interaction produces periodically pulsating harmonic torques. Although their average value is zero, they cause acoustic noise. Increasingly, mechanical resonance and vibration may occur. In the case $8 \mathrm{P} 1$, the fundamental and all harmonics form 8-pole MMF waves, whereas in the case 4P2, they form 4-pole MMF waves. The pulsating harmonic torque resulting from the interaction between the fundamental air-gap flux and the $(3 n-1)$ th rotor harmonic MMF wave is given by:

$T_{3 n-1}=\frac{Z_{r} p}{2 \sqrt{2}} \phi_{1} I_{r, 3 n-1} \cos \left(\theta_{3 n-1}-3 n \omega t\right)$

where $Z_{r}$ is the number of slots in rotor, $p$ is pole pairs, $\phi_{1}$ is the fundamental flux, $I_{r, 3 n-1}$ is the (3n-1)th rotor harmonic current, $\theta_{3 n-1}$ is the angle by which the (3n-1)th harmonic voltage leads the fundamental voltages, as shown in Fig. 12(a).

For the $(3 n+1)$ th harmonic, the pulsating harmonic torque is calculated by:

$T_{3 n+1}=-\frac{Z_{r} p}{2 \sqrt{2}} \phi_{1} I_{r, 3 n+1} \cos \left(\theta_{3 n+1}+3 n \omega t\right)$

where $I_{r, 3 n+1}$ is the $(3 n+1)$ th rotor harmonic current, $\theta_{3 n+1}$ is the angle by which the $(3 n+1)$ th harmonic voltage leads the fundamental voltages, as shown in Fig. 12(b).

In the case 8P2, the fundamental and odd order harmonics are in phase and form 8-pole MMF waves, whereas the even order harmonics are out of phase and form 4-pole ones. Thus, (12) and (13) can be used to calculate the pulsating harmonic torques due to the interaction between the fundamental airgap flux and the odd order harmonic currents. For the even order harmonics, the pulsating harmonic torques arising from the interaction of the fundamental air-gap flux and the (3n1)th rotor harmonic currents can be calculated by:

$$
\begin{aligned}
T_{3 n-1}^{8 P 2, e}= & \frac{Z_{r} p_{8}}{2 \sqrt{2}} \frac{1}{\pi} \phi_{1} I_{r, 3 n-1}\left\{2 \sin \left(3 n \omega t-\theta_{3 n-1}\right)\right. \\
& \left.+\frac{2}{3} \sin \left[(3 n-2) \omega t-\theta_{3 n-1}\right]\right\}
\end{aligned}
$$

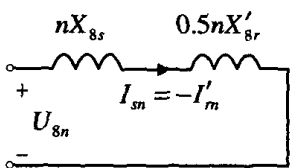

(a)

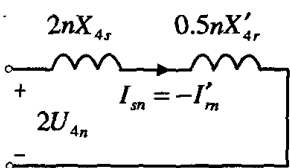

(b)
Fig.9. Simplified equivalent circuits. (a) 4-pole operation. (b) 8-pole operation.
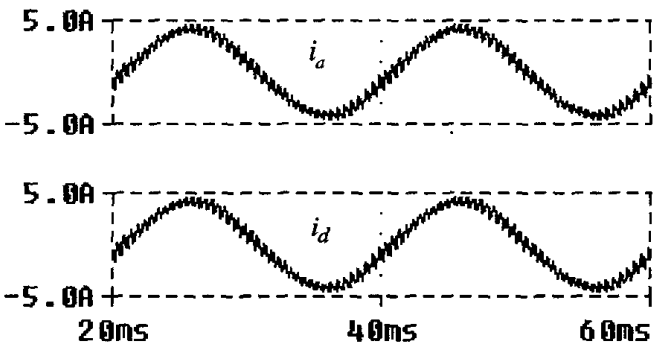

(a)
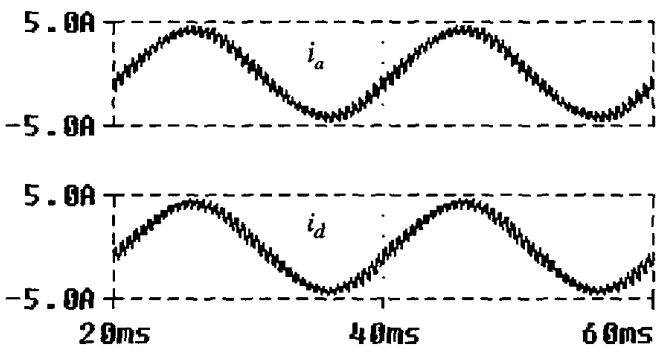

(b)

Fig. 10. Current waveforms of windings a and d. (a) 8P1. (b) $8 \mathrm{P} 2$.
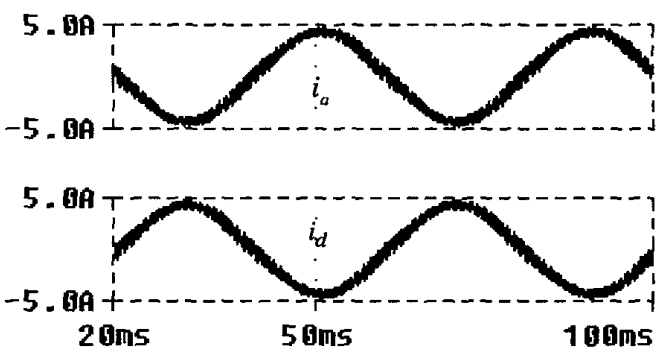

(a)
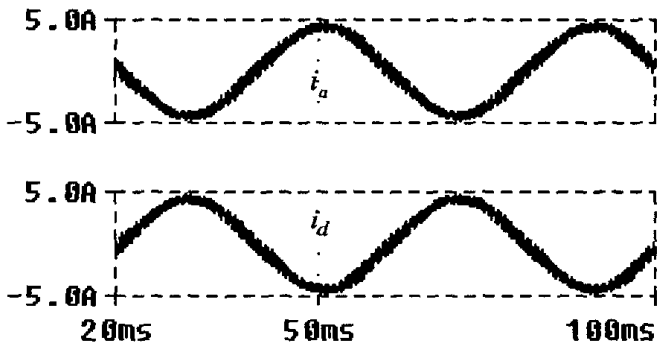

(b)

Fig. 11. Current waveforms of windings a and d. (a) $4 \mathrm{P} 1$. (b) $4 \mathrm{P} 2$ 


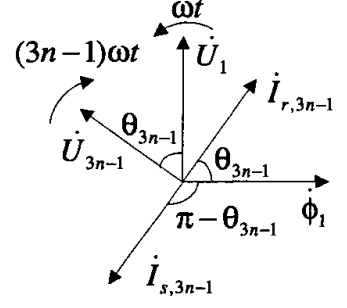

(a)

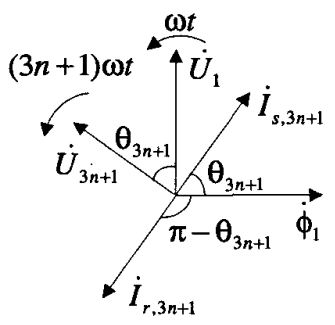

(b)
Fig. 12. The phasor diagrams. (a) Negative sequence.

(b) Positive sequence.

where $p_{8}$ is equal to 4 and the superscript $8 P 2$,e represents the case $8 \mathrm{P} 2$ and even order harmonic. The pulsating harmonic torques arising from the interaction of the fundamental air-gap flux and the $(3 n+1)$ th rotor harmonic currents can be calculated by:

$$
\begin{aligned}
T_{3 n+1}^{8 P 2, e}= & \frac{Z_{r} p_{8}}{2 \sqrt{2}} \frac{1}{\pi} \phi_{1} I_{r, 3 n+1}\left\{2 \sin \left(3 n \omega t+\theta_{3 n+1}\right)\right. \\
& \left.+\frac{2}{3} \sin \left[(3 n+2) \omega t+\theta_{3 n+1}\right]\right\}
\end{aligned}
$$

In the case 4P1, the fundamental and odd order harmonics are out of phase and form 4-pole MMF waves, whereas the even order harmonics are in phase and form 8-pole ones. Similar to the case $8 \mathrm{P} 2,(12)$ and (13) can also be used to calculate the pulsating harmonic torques due to the interaction between the fundamental air-gap flux and the odd order harmonic currents. For the even order harmonics, the pulsating harmonic torques arising from the interaction of the fundamental air-gap flux and the (3n-1)th rotor harmonic currents can be calculated by:

$$
\begin{aligned}
T_{3 n-1}^{4 P 1, e}= & \frac{Z_{r} p_{4}}{2 \sqrt{2}} \frac{1}{\pi} \phi_{1} I_{r, 3 n-1}\left\{-2 \sin \left(3 n \omega t-\theta_{3 n-1}\right)\right. \\
& \left.+\frac{2}{3} \sin \left[(3 n-2) \omega t-\theta_{3 n-1}\right]\right\}
\end{aligned}
$$

where $p_{4}$ is equal to 2 , the superscript $4 P 1, e$ represents the case $4 \mathrm{P} 1$ and even order harmonic. The pulsating harmonic torques arising from the interaction of the fundamental flux and the $(3 n+1)$ th rotor harmonic currents can be calculated by:

$$
\begin{aligned}
T_{3 n+1}^{4 P 1, e}= & \frac{Z_{r} p_{4}}{2 \sqrt{2}} \frac{1}{\pi} \phi_{1} I_{r, 3 n+1}\left\{-2 \sin \left(3 n \omega t+\theta_{3 n+1}\right)\right. \\
& \left.+\frac{2}{3} \sin \left[(3 n+2) \omega t+\theta_{3 n+1}\right]\right\}
\end{aligned}
$$

In general, the vibration and acoustic noise are generated by those pulsating torques of high frequencies, and their intensities are proportional to the magnitude of the pulsating torque [6]. From the voltage amplitude spectrum shown in Fig. 6, it can be found that the harmonic currents with frequencies at the integer multiples of the carrier frequency produce the maximum noise. This is because the hearing is sensitive to these frequencies, and the magnitudes of harmonic currents at these frequencies are large. Consider the $(3 \mathrm{n}-1)$ th rotor harmonic current in the case $8 \mathrm{P} 1$, the frequency of the pulsating harmonic torque expressed in (12) is $3 \mathrm{n}$ multiples of the fundamental frequency. But, in the case $8 \mathrm{P} 2$, the torque expressed in (14) consists of two components, one is still $3 \mathrm{n}$ times the fundamental frequency, another is $3 \mathrm{n}$ 1 times the fundamental frequency. Compared with the carrier frequency, the fundamental frequency is low. The effects of two composites on hearing are the same. In addition, the maximum value of (12) is 1.176 times that of (14). For the $(3 n+1)$ th rotor harmonic current, the same result can be obtained. Therefore the acoustic noise in the case 8P2 is less than that in the case $8 \mathrm{P} 1$. For the same reason, the acoustic noise in the case 4P1 is less than that in the case 4P2.

In view of reducing the acoustic noise, the cases $8 \mathrm{P} 2$ and 4P1 are more suitable to pole-changing induction motor drives.

\section{CONCLUSION}

A new dual-inverter pole-changing SCIM drive has been proposed which has the advantages of high-speed constantpower operation and suppressed harmonic torques (hence suppressed acoustic noise). The coil pitch is optimized that it not only makes the maximum torques at both pole pairs as large as possible, but also realizes the large maximum torque difference between two different pole pairs. The key of reducing acoustic noise is to purposely select that the two carriers of the dual inverters are exactly out of phase for the 8-pole operation whereas the two carriers are in phase during the 4-pole operation.

\section{ACKNOWLEDGMENT}

The work was supported in part by CRCG of the University of Hong Kong and the Hong Kong Research Grants Council. The authors would like to thank Dr. Z.Q. Zhu for his valuable information.

\section{REFERENCES}

[1] C.C. Chan, "An overview of electric vehicle technology," IEEE Proc., vol. 81, no. 9, 1993, pp. 1202-1213.

[2] T. Mizuno, K. Tsuboi, I Hirotsuka, S. Suzuki, I. Matsuda, and T. Kobayashi, "Basic principle and maximum torque characteristics of a six-phase pole change induction motor for electric vehicles," Electrical Engineering in Japan, vol. 118, no. 3, 1997, pp. 78-91.

[3] A.M. Trzynadlowski, F. Blaabjerg, J.K. Pedersen, R.L. Kirlin, and S. Legowski, "Random pulse width modulation techniques for converterfed drive systems - a review," IEEE Trans. Industry Applicant., vol. 30, no. 5, 1994, pp. 1166-1175.

[4] Z.Q. Zhu and D. Howe, "Improved methods for prediction of electromagnetic noise radiated by electric machines," IEE Procedings Electric Power Applications, vol. 141, no. 2, 1994, pp. 109-120.

[5] G.K. Dubey, Power Semiconductor Controlled Drives. Prentice Hall, 1989.

[6] D.Z. Xu, The Theory of Variable Speed for AC Motor. Zhejiang University Press, 1991. 